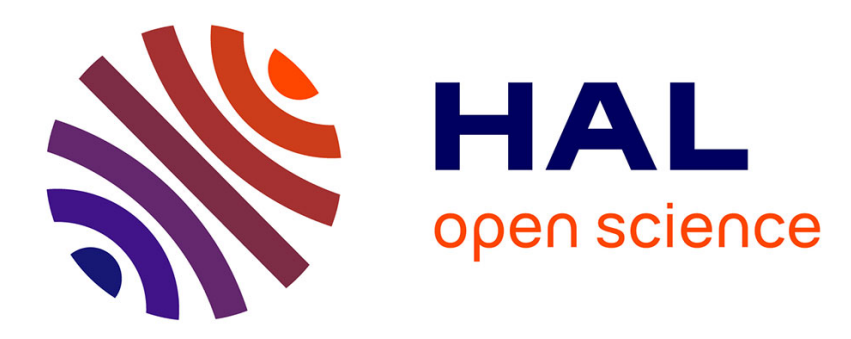

\title{
Doppler effect as a tool for ultrashort electric field reconstruction
}

P Béjot, E Szmygel, A Dubrouil, F Billard, B Lavorel, O Faucher, E Hertz

\section{To cite this version:}

P Béjot, E Szmygel, A Dubrouil, F Billard, B Lavorel, et al.. Doppler effect as a tool for ultrashort electric field reconstruction. Optics Letters, 2020, 45 (24), pp.6795-6798. 10.1364/OL.402935 . hal02998500

\section{HAL Id: hal-02998500 \\ https://hal.science/hal-02998500}

Submitted on 10 Nov 2020

HAL is a multi-disciplinary open access archive for the deposit and dissemination of scientific research documents, whether they are published or not. The documents may come from teaching and research institutions in France or abroad, or from public or private research centers.
L'archive ouverte pluridisciplinaire HAL, est destinée au dépôt et à la diffusion de documents scientifiques de niveau recherche, publiés ou non, émanant des établissements d'enseignement et de recherche français ou étrangers, des laboratoires publics ou privés. 


\title{
Doppler effect as a tool for ultrashort electric field reconstruction.
}

\author{
P. BÉJot ${ }^{1}$, E. Szmygel ${ }^{1,2}$, A. Dubrouil ${ }^{2}$, F. Billard ${ }^{1}$, B. LAVorel ${ }^{1}$, O. FAucher ${ }^{1}$, ANd E. \\ HERTZ ${ }^{1}$, \\ *edouard.hertz@u-bourgogne.fr \\ ${ }^{1}$ Laboratoire Interdisciplinaire Carnot de Bourgogne, UMR CNRS 6303 - Université Bourgogne Franche-Comté, 21078 Dijon Cedex, France \\ ${ }^{2}$ Femto Easy, Batiment Gienah, Cité de la Photonique, 11 avenue de Canteranne, 33600 Pessac France.
}

Compiled November 7, 2020

\begin{abstract}
We present a new variant of spectral-shearing interferometry method for characterizing ultrashort laser pulses. This original approach, called Doppler Effect E-field Replication (DEER), exploits the rotational Doppler effect for producing the frequency shear and provides a spectral-shearing in the absence of frequency conversion enabling operation in the ultraviolet spectral range. Evaluation of the DEER-SPIDER set-up reveals a phase reconstruction of great reliability. Possible improvements, benefits, and worthwhile prospects of the method are discussed. (๑ 2020 Optical Society of America
\end{abstract}

OCIS codes: (190.7110) Ultrafast nonlinear optics; (260.5430) Polarization; (320.7080) Ultrafast devices; (320.0320) Ultrafast optics; (320.7100) Ultrafast measurements.

\section{http://dx.doi.org/10.1364/ao.XX.XXXXXX}

The generation of ultrashort pulses has known during the last decades major technological breakthroughs with the emergence of new coherent sources in the infrared (IR) and ultraviolet (UV), the generation of super-continua [1], IR [2] or Terahertz [3] nearsingle cycle pulses, and XUV attosecond pulses [4]. Theses developments have been a driving element for spectacular advances. The utilization of such pulses requires a comprehensive characterization of the electric field that led to a growing interest in reliable and versatile diagnostic tools $[5,6]$. Nowadays, the two most widespread self-referenced complete characterization methods are the frequency-resolved optical gating [6, 7] (FROG) and the spectral phase interferometry for direct electric field reconstruction $[6,8,9]$ (SPIDER), while other approaches like d-scan [10] or SRSI [11, 12] are of growing interest. The approach described in the present letter can be seen as a variant of the SPIDER. The SPIDER relies on the spectral interferometry between the pulse to be characterized and its replica that is both spectrally and temporally shifted. The interferogram yields the gradient of the spectral phase from which the spectral phase can be retrieved. The frequency shift or shear $\Omega$ is produced by a nonlinear effect (usually sum frequency mixing) between a sequence of two time-delayed pulses and a highly chirped pulse. Spectral-shearing interferometry techniques as SPIDER feature several advantages. Among them, they provide a selfreferenced measurement under single-shot operation and the inversion procedure to recover the spectral phase is straightforward, non-iterative, and unambiguous since it relies on Fourier transforms and spectral filtering. The SPIDER is also rather insensitive to the spectral dependence of the nonlinear optical process and to the noise [9] since the relevant information is encoded into the fringes spacing of the interferogram and not in the spectral amplitude. Since the first demonstration of SPIDER [8], considerable efforts have been made to extend the scope of measurements $[6,9]$ giving birth to several variants of the original design enabling for instance the measurements of weak pulses [13], of spatio-temporal distortions [14], or measurements free from phase distortions induced by the beamsplitter [15]. In all theses variants, the shear is produced by sum-frequency generation. The absorption and phase matching bandwidth of crystals (as well as absorption bands of oxygen for harmonic signals lying in the vacuum UV) therefore limit the functionality of standard devices for the characterization of UV laser pulses. While variants of d-scan [16] or FROG [17, 18] have been applied for characterizing UV pulses, there is still a need for methods enabling self-referenced and single-shot measurement of weak pulses as intrinsically provided by SPIDER devices. In specific cases, the shear can be produced in non-standard ways from gold surfaces [19] or in gas [20]. A scheme called DC-SPIDER [15, 21], relying on the difference frequency generation (DFG) between the pulse to characterize and a chirped pulse of smaller frequency, has also been proposed for the characterization of UV pulses. Nevertheless, the DC-SPIDER (as other methods based on DFG like DFG XFROG [18]) is not strictly speaking self-consistent and the need of two input beams at different frequencies limits the use of this approach to custom developments. Another method called SD-SPIDER has also been reported [22]. Unlike most variants of the SPIDER, the SD-SPIDER is based on a third-order nonlinear optical process, namely self-diffraction (SD) effect, rather than sum-frequency generation. A spectral-shearing interferometry method based on SD is in principle well suited for the characterization of UV or broadband laser pulses since it operates at the fundamental wavelength and does not acquire frequency conversion. Unfortunately, the SD process is not phase-matched and the device does not measure the phase of the pulse but that of its second harmonic requiring an elaborate retrieval procedure. Be- 
sides these drawbacks, the use of a third-order nonlinear effect through spectral interferometry appears very desirable. To this end, new concepts have to be considered and we present here an original approach exploiting the rotational Doppler frequency shift [23-25] that lifts the inherent flaws of the SD phenomenon. As detailed below, the overall mechanism relies on a third-order nonlinear optical effect, perfectly phase-matched, and providing a direct phase retrieval with an extended functionality toward UV (spectral domain of importance since many organic systems have their absorption bands in this range). The present method, called Doppler Effect E-field Replication (DEER) SPIDER is evaluated for the characterization of short UV laser pulses (40 fs duration, $\lambda=350 \mathrm{~nm}$ ) for which the second harmonic would lie in the vacuum UV.

As already mentioned, the SPIDER consists of measuring the interferogram associated to a sequence of two pulses delayed by $\tau$ and spectrally shifted by $\Omega$. Writing $\omega$ the angular frequency and $|E(\omega)| e^{i \varphi(\omega)}$ the complex electric field to be characterized (expressed in the frequency-domain), the interferogram writes as [8]:

$$
\begin{aligned}
S(\omega) & =|E(\omega)|^{2}+|E(\omega+\Omega)|^{2} \\
& +2|E(\omega)||E(\omega+\Omega)| \cos (\omega \tau+\Gamma(\omega)),
\end{aligned}
$$

where $\Gamma(\omega)=\varphi(\omega+\Omega)-\varphi(\omega)$ contains the phase $\varphi(\omega)$ to be recovered. The relevant information is encoded in the shape of the fringes. With no chirp $[\Gamma(\omega)=0]$ the argument of the cosine reduces to $\omega \tau$ and the fringes present an equal frequency spacing inversely proportional to the delay $\tau$. Any change of this interferogram (spreading or shrinking) reflects the presence of a chirp. The cosine argument can be extracted through spectral filtering and the knowledge of $\tau$ allows the evaluation of $\Gamma(\omega)$ that is proportional to the spectral phase derivative. The spectral phase $\varphi(\omega)$ of the pulse can thus be retrieved (up to a constant) by integrating $\Gamma(\omega)$ [6]. The central key of the procedure is the production of the frequency shear $\Omega$ and we propose in this issue to exploit the rotational Doppler effect which is the rotational counterpart of the well-known translational Doppler effect. The rotational Doppler effect takes place for instance when a circularly polarized (CP) light interacts with an anisotropic object in rotation (in the plane of the field polarization). A frequencyshifted counter-rotating wave is then produced. This last is shifted by $\Delta \omega= \pm 2 \Omega_{\text {rot }}$, where $\Omega_{\text {rot }}$ stands for the angular frequency of the anisotropic object and where the sign of the shift depends on the helicity of the CP wave with respect to the sense of rotation of the object. The frequency is downshifted (upshifted) for an incident CP field rotating in the same (opposite) sense as the object. The spectrally shifted counter-rotating wave is accompanied by an unshifted co-rotating wave. The same occurs with the translational Doppler effect where a wave interacting with a semi-transparent object gives rise to a spectrally shifted reflected wave with an unshifted transmitted one. Rotational doppler shifts have been observed with anisotropic optical elements in mechanical rotation [26] or with rotating molecules $[24,25]$. Here we show that the rotational Doppler effect can also be applied for spectral-shearing interferometry. In this issue, the frequency shear should be a reasonable portion of the pulse's spectral bandwidth, in practice about a tenth of the spectral bandwidth is well-suited. In our case, this corresponds to a shear $\Delta \lambda \approx 0.5 \mathrm{~nm}$ at $350 \mathrm{~nm}$ requiring an angular speed of the anisotropy $\Omega_{\text {rot }}$ in the range of $10^{12} \mathrm{rad} / \mathrm{s}$. Needless to say that such a rotating anisotropy cannot be reached by a mechanical rotation. Instead, our strategy relies on a nonlinear optical techniques and more precisely on the third-order instantaneous electronic Kerr effect. A linearly polarized field interacting with a piece of glass induces a birefringence proportional to the square of the applied electric field. The material acts therefore as a wave plate with neutral axes along and perpendicular to the field polarization. The requisite rotation of anisotropy, for our purpose of rotational Doppler shift, is produced by means of a pulse of twisted linear polarization (TLP), namely a pulse with a linear polarization that continuously rotates at a speed in the $\mathrm{THz}$ range. Such a polarization shaping has been successfully applied to induce unidirectional rotational motion of linear molecules [25]. The main part of the DEER-SPIDER set-up, implemented for the characterizing UV pulses, is depicted in Fig. 1. The UV pulses ( $40 \mathrm{fs}$ duration, $\lambda=350 \mathrm{~nm}$ ) are delivered by a Ti:sapphire laser equipped with a NOPA (noncollinear optical parametric amplifier). The input pulse (horizontally polarized)

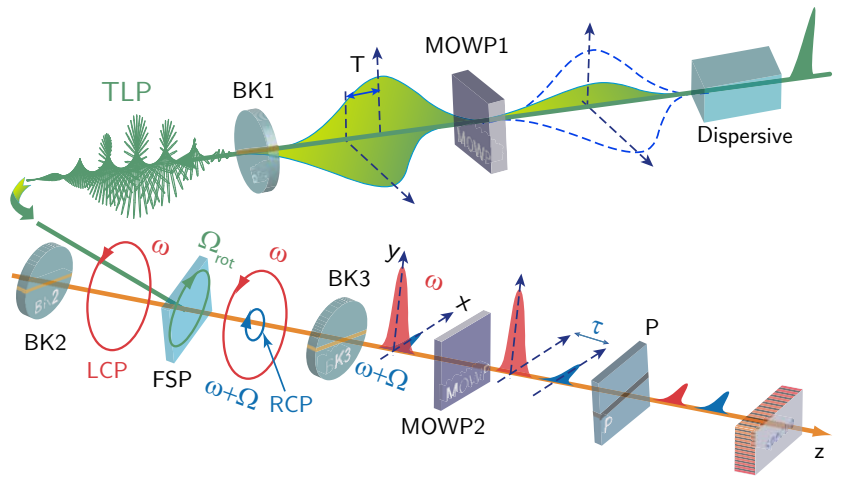

Fig. 1. Schematic representation of the experimental set-up. MOWP: multiple order wave plate, BK: Berek compensator, TLP: pulse of twisted linear polarization, LCP (RCP) left (right) circular polarization, FSP: fused Silica plate, P: polarizer.

is split into two parts. One is used to produce the TLP pulse and the other one contains the "reference pulse" to analyze. Different strategies can be implemented for the production of ultrashort TLP pulses [27]. We have selected a method that relies on the production of two counter-rotating CP and time-delayed chirped pulses. Basically, a field of spinning linear polarization can be seen as the superposition of two CP fields with slightly different frequencies and opposite handedness. Our approach, depicted in Fig. 1 (top part), is based on this principle. The TLP pulse is created by generating two orthogonally polarized chirped pulses that are time-delayed by $T, T$ being small enough to insure a significant overlap between the two pulses. To that end, the input pulse is first temporally chirped via a thick dispersive medium inducing a chirp $\Phi^{\prime \prime} \approx 24000 \mathrm{fs}^{2}$. The pulse then propagates through a multiple order wave plate (MOWP1) providing the sequence of two time-delayed and cross polarized fields. A Berek compensator (BK1) transforms the two perpendicular components into two $\mathrm{CP}$ fields of opposite helicity. Each pulse presents a frequency drift with the time so that the delay $T$ between them is equivalent to have an effective constant frequency shift. The configuration is therefore analog to the sum of two spectrally-shifted CP fields of opposite handedness resulting in the desired TLP pulse. It has been shown [27] that for a Gaussian pulse shape the field vector rotates with a temporal period $T_{\text {rot }}=2 \pi /(a T)$ where $a$ depends on the quadratic 
spectral phase $\Phi^{\prime \prime}$ :

$$
a=\frac{\Phi^{\prime \prime}}{\frac{\Delta t^{4}}{(2 \sqrt{2} \ln 2)^{2}}+2 \Phi^{\prime \prime 2}}
$$

with $\Delta t$ the FTL pulse duration. The main advantage of this strategy, in comparison with the one implemented so far for producing spinning molecules [25], relies on the production of long TLP pulses with potentially many turns of the electric field [see Fig. 1]. The angular Doppler effect is therefore less sensitive to the temporal synchronization between the TLP pulse and the pulse to be spectrally shifted. Furthermore, the production of long TLP pulses is more immune with respect to the input chirp and allows the characterization of larger spectral phases. Finally, for large values of $\Phi^{\prime \prime}$, Eq. 2 gives $a \approx 1 /\left(2 \Phi^{\prime \prime}\right)$ so that $a$ and therefore the shear does not depend on the incident pulse bandwidth to be measured. In our case, the pulse of typical energy $2 \mu \mathrm{J}$ is stretched to about $\Delta t_{\mathrm{ch}}=1.7 \mathrm{ps}\left(\Phi^{\prime \prime}=24000 \mathrm{fs}^{2}\right)$ and the delay is fixed to $T=190 \mathrm{fs}$. The rotational period of the spinning polarization is $T_{\text {rot }}=1.59 \mathrm{ps}$ providing a calculated shear $\Delta \lambda \approx 0.51 \mathrm{~nm}(\Omega=7.85 \mathrm{rad} / \mathrm{ps})$.

The reference pulse, for its part, is time-delayed with a delay line and $\mathrm{CP}$ by passing through a Berek compensator [BK2 in Fig. 1 lower part]. The use of Berek compensators as polarizing components offers more flexibility than waveplates via a variable dephasing and induces smaller dispersion (about 120 $\mathrm{fs}^{2}$ at $350 \mathrm{~nm}$ ). Reference and TLP pulses, temporally synchronized, are focused by the same lens (focal length $f=15 \mathrm{~cm}$ ) into a $1 \mathrm{~mm}$ fused silica plate with a small angle $\left(\approx 4^{\circ}\right)$. The TLP pulse induces a rotating birefringence within the plate, and the field to be characterized contains after the interaction a spectrally-shifted CP field of opposite handedness $E(\omega+\Omega)$ [or $E(\omega-\Omega)]$ together with a stronger unshifted CP field of same helicity $E(\omega)$ [Fig. 1]. The spectral interferometry is conducted between these two components. The TLP beam is blocked while the reference pulse passes through a Berek compensator (BK3). The two CP components are converted into linearly polarized fields of crossed polarizations. They are further time-delayed by $\tau=1.45$ ps by means of a multiple order wave plate (MOWP2). A polarizer $(\mathrm{P})$ is then orientated to balance their amplitudes so as to maximize the visibility of the spectral fringes measured by a spectrometer. The condition of two time-delayed sheared pulses (but otherwise identical) for spectral shearing interferometry is therefore fulfilled.

The first point to be checked is the ability of our device to generate suitable frequency shear $\Omega$ which constitutes the indispensable prerequisite for our purpose of pulse characterization. This shear has been measured by inverting the sens of rotation of the TLP pulse via BK1 so as to shift the observed signal from $\Omega$ to $-\Omega$. The two spectra shown in Fig. 2(a) are found to be shifted by $\Delta \omega \approx 2 \Omega=16.0 \mathrm{rad} / \mathrm{ps}$ indicating a frequency shear $\Omega=8.0 \mathrm{rad} / \mathrm{ps}(\Delta \lambda \approx 0.52 \mathrm{~nm})$ in excellent agreement with the features of the TLP pulse produced by our polarization shaping. This undoubtedly points out the ability of the rotational Doppler effect to generate suitable shears for the characterization of fs laser pulse. When the polarizer $(\mathrm{P})$ is turned so as to maximize the contrast of spectral fringes, the interferogram between the two components can be observed as shown in Fig. 2(b). The information about the spectral phase is in principle imprinted into the fringes pattern through the deviations from an equal frequency spacing. Black line in Fig. 2(c) depicts the DEERSPIDER characterization of the pulse as delivered by the NOPA. A small resulting cubic phase, not compensated in the laser,

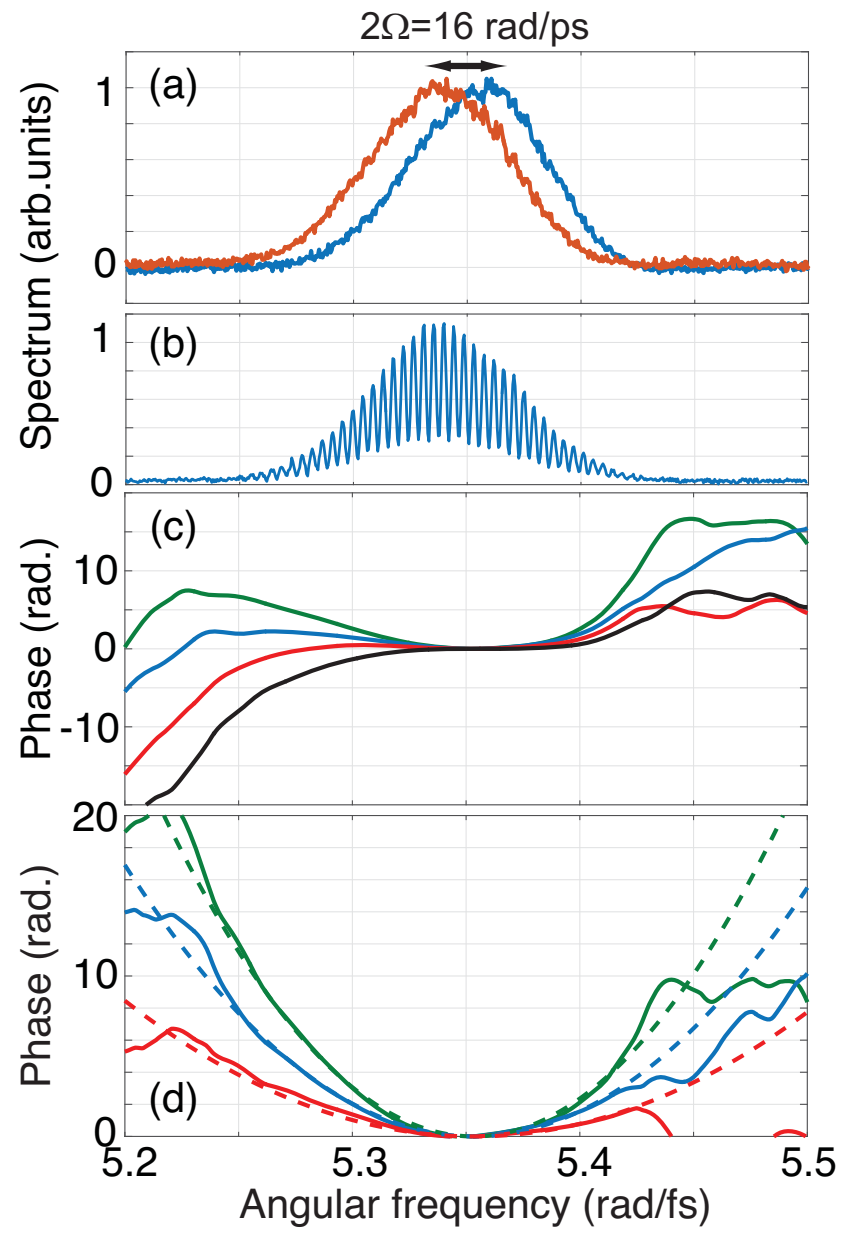

Fig. 2. (a) Frequency shear measured by inverting the sens of rotation of the TLP pulse. (b) Interferogram. (c) Spectral phases of a UV pulse retrieved from the DEER-SPIDER. The black line corresponds to the phase of the laser output and color lines to the phases after propagation through different fused silica (FS) plates (red, blue, green $=6,12,18 \mathrm{~mm}$ thicknesses). (d) Contribution from FS plates in the measured spectral phases (solid lines) compared to the FS second-order dispersion (dashed lines).

can be clearly distinguished. In order to validate our method, calibrated chirps produced by fused silica (FS) plates of different thicknesses have been introduced. Measurements depicted in red, blue and green in Fig. 2(c) reveal the emergence of a quadratic phase contribution above the initial cubic phase. By subtracting the input phase, the phase contributions from the FS plates can be isolated as shown in Fig. 2(d). The observed quadratic phases are then compared to the calculated secondorder dispersion and are found in very good agreement with an RMS error field $\epsilon \leq 0.1$. This observation is a clear evidence that the present approach is operating in the UV spectral range and provides a reliable characterization of ultrafast UV laser sources on a single-shot basis. We emphasize that the method has also been successfully applied for characterizing complex IR pulse shapes produced by a pulse shaper device [28].

The present letter describes the first proof-of-principle of a new method. The technique could be further improved. For instance, special efforts in spectral shearing techniques have been 
devoted to avoid the dispersion mismatch introduced by the beamsplitter used to produce the pair of pulses. In the present case, this effect is limited since the pulse separation is performed after the interaction. The dispersion mismatch originates only from the differential dispersion introduced by MOWP2 (about $270 \mathrm{fs}^{2}$ ) that could be significantly reduced by using a MgF2 MOWP. The dispersion mismatch would then be reduced to $50 \mathrm{fs}^{2}$ and $5 \mathrm{fs}^{2}$ for a delay $\tau=1$ ps and $100 \mathrm{fs}$, respectively. Furthermore, the dispersion onto the reference pulse introduced by BK2 before the interaction could also be avoided by producing the $\mathrm{CP}$ through metallic reflexion. One challenge, in the topic of pulse characterization, is to find alternative nonlinear interactions for encoding in a robust way the spectral phase of short laser pulses with exotic wavelengths. We believe that the rotational Doppler effect appears as a promising approach in this pursued objective as well as for other applications. This effect can for instance be exploited to tune the wavelength of laser fields. In this context, the use of broadband radiations, such as super-continua, to produce the TLP pulse should allow an ultrafast rotation of the field vector inducing controllable giant frequency shift (about $\sqrt{2}$ times the bandwidth of the TLP pulse [27]). Rotational Doppler effect could also be of interest to control the wavelengths of $\mathrm{THz}$ fields not otherwise easily accessible.

\section{FUNDING}

This work was supported by the CNRS, the ERDF Operational Programme - Burgundy, the EIPHI Graduate School (Contract No. ANR-17-EURE-0002). The PhD of E.S. is funded by a CIFRE contract $(2018 / 0253)$ granted by ANRT. Calculations were performed using HPC resources from DNUM-CCUB (Université de Bourgogne).

\section{DISCLOSURES}

ES,AD: Femto Easy(E). The authors declare no conflicts of interest.

\section{REFERENCES}

1. R. R. Alfano and S. L. Shapiro, "Observation of self-phase modulation and small-scale filaments in crystals and glasses," Phys. Rev. Lett. 24, 592-594 (1970).

2. G. Krauss, S. Lohss, T. Hanke,et al. "Synthesis of a single cycle of light with compact erbium-doped fibre technology," Nat. Photon. 4, 33-36 (2010)

3. H. Hirori, A. Doi, F. Blanchard, and K. Tanaka, "Single-cycle terahertz pulses with amplitudes exceeding $1 \mathrm{MV} / \mathrm{cm}$ generated by optical rectification in $\mathrm{LiNbO}_{3}$," Appl. Phys. Lett. 98, 091106- (2011).

4. F. Calegari, G. Sansone, S. Stagira, C. Vozzi, and M. Nisoli, "Advances in attosecond science," J. Phys. B: At. Mol. Opt. Phys. 49, 062001 (2016).

5. A. Monmayrant, S. Weber, and B. Chatel, "A newcomer's guide to ultrashort pulse shaping and characterization," J. Phys. B: At. Mol. Opt. Phys. 43, 103001- (2010).

6. I. A. Walmsley and C. Dorrer, "Characterization of ultrashort electromagnetic pulses," Adv. Opt. Photon 1, 308-437- (2009).

7. R. Trebino, K. W. DeLong, D. Fittinghoff, J. Sweetser, M. A. Krumbügel, B. Richman, and D. Kane, "Measuring ultrashort laser pulses in the time-frequency domain using frequency-resolved optical gating," Rev. Sci. Instruments 68, 3277-3295 (1997).
8. C. laconis and I. A. Walmsley, "Spectral phase interferometry for direct electric-field reconstruction of ultrashort optical pulses," Opt. Lett. 23, 792-794 (1998).

9. M. E. Anderson, A. Monmayrant, S.-P. Gorza, P. Wasylczyk, and I. A. Walmsley, "Spider: A decade of measuring ultrashort pulses," Laser Phys. Lett. 5, 259-266- (2008).

10. M. Miranda, T. Fordell, C. Arnold, A. L'Huillier, and H. Crespo, "Simultaneous compression and characterization of ultrashort laser pulses using chirped mirrors and glass wedges," Opt. Express 20, 688-697 (2012).

11. T. Oksenhendler, S. Coudreau, , N. Forget, et al. "Self-referenced spectral interferometry," Appl. Phys. B 99, 7-12 (2010).

12. S. Grabielle, S. Coudreau, V. Crozatier, N. Forget, et al. "Selfreferenced spectral interferometry in the UV domain," Eur. EQEC 2011 Conf. Dig. , OSA Technical Digest (CD), paper CF p22 (2011).

13. M. Hirasawa, N. Nakagawa, K. Yamamoto, R. Morita, H. Shigekawa, and M. Yamashita, "Sensitivity improvement of spectral phase interferometry for direct electric-field reconstruction for the characterization of low-intensity femtosecond pulses," Appl. Phys. B 74, s225-s229 (2002).

14. E. M. Kosik, A. S. Radunsky, I. A. Walmsley, and C. Dorrer, "Interferometric technique for measuring broadband ultrashort pulses at the sampling limit," Opt. Lett. 30, 326-328 (2005).

15. P. Baum, S. Lochbrunner, and E. Riedle, "Zero-additional-phase spider: full characterization of visible and sub-20-fs ultraviolet pulses," Opt. Lett. 29, 210-212 (2004).

16. A. Tajalli, T. Kalousdian, M. Kretschmar, S. Kleinert, U. Morgner, and T. Nagy, "Full characterization of 8 fs deep UV pulses via a dispersion scan," Opt. Lett. 44, 2498-2501 (2019).

17. T. Nagy and P. Simon, "Single-shot TG FROG for the characterization of ultrashort DUV pulses," Opt. Express 17, 8144-8151 (2009).

18. S. Linden, J. Kuhl, and H. Giessen, "Amplitude and phase characterization of weak blue ultrashort pulses by downconversion," Opt. Lett. 24, 569-571 (1999)

19. M. E. Anderson, T. Witting, and I. A. Walmsley, "Gold-SPIDER: spectral phase interferometry for direct electric field reconstruction utilizing sum-frequency generation from a gold surface," J. Opt. Soc. Am. B 25, A13-A16 (2008).

20. A. J. Verhoef, A. Mitrofanov, A. Zheltikov and A. Baltuška, "Plasmablueshift spectral shear interferometry for characterization of ultimately short optical pulses," Opt. Lett. 34, 82-84 (2009).

21. P. Londero, M. E. Anderson, C. Radzewicz, C. laconis, and I. A. Walmsley, "Measuring ultrafast pulses in the near-ultraviolet using spectral phase interferometry for direct electric field reconstruction," J. Mod. Opt. 50, 179-184 (2003).

22. S. Birkholz, G. Steinmeyer, S. Koke, D. Gerth, S. Bürger, and B. Hofmann, "Phase retrieval via regularization in self-diffraction-based spectral interferometry," J. Opt. Soc. Am. B 32, 983-992 (2015).

23. B. A. Garetz, "Angular doppler effect," J. Opt. Soc. Am. 71, 609-611 (1981).

24. O. Korech, U. Steinitz, R. Gordon, I. Sh. Averbukh, and Y. Prior, "Observing molecular spinning via the rotational doppler effect," Nat. Photonics 7, 711-714 (2013).

25. G. Karras, M. Ndong, E. Hertz, D. Sugny, F. Billard, B. Lavorel, and O. Faucher, "Polarization shaping for unidirectional rotational motion of molecules," Phys. Rev. Lett. 114, 103001- (2015).

26. B. A. Garetz and S. Arnold, "Variable frequency shifting of circularly polarized laser radiation via a rotating half-wave retardation plate," Opt. Commun. 31, 1-3 (1979).

27. O. Faucher, E. Hertz, B. Lavorel, and F. Billard, "Linear and nonlinear optics in coherently spinning molecules," in Progress in Ultrafast Intense Laser Science XIV, vol. 118 of Springer Series in Chemical Physics, 2018, 37-64

28. P. Béjot et al, "in preparation". 